\title{
Genomics analysis of genes encoding respiratory burst oxidase homologs (RBOHs) in jatropha and the comparison with castor bean
}

\author{
Yongguo Zhao ${ }^{1,2}$, Zhi Zou ${ }^{\text {Corresp. } 2}$ \\ ${ }^{1}$ Guangdong University of Petrochemical Technology, Maoming, Guangdong, China \\ 2 Hainan Key Laboratory for Biosafety Monitoring and Molecular Breeding in Off-Season Reproduction Regions, Key Laboratory of Biology and Genetic \\ Resources of Tropical Crops, Ministry of Agriculture and Rural Affairs, Institute of Tropical Biosciences and Biotechnology, Chinese Academy of Tropical \\ Agricultural Sciences, Haikou, Hainan, China \\ Corresponding Author: Zhi Zou \\ Email address: zouzhi@itbb.org.cn
}

Respiratory burst oxidase homologs (RBOHs), which catalyze the production of superoxide from oxygen and NADPH, play key roles in plant growth and development, hormone signaling, and stress responses. Compared with extensive studies in model plants arabidopsis and rice, little is known about RBOHs in other species. This study presents a genome-wide analysis of Rboh family genes in jatropha (Jatropha curcas) as well as the comparison with castor bean (Ricinus communis), another economically important nonfood oilseed crop of the Euphorbiaceae family. The family number of seven members identified from the jatropha genome is equal to that present in castor bean, and further phylogenetic analysis assigned these genes into seven groups named RBOHD, $-C,-B,-E$, $\mathrm{F},-\mathrm{N}$, and $-\mathrm{H}$. In contrast to a high number of paralogs present in arabidopsis and rice that experienced several rounds of recent whole-genome duplications, no duplicate was identified in both jatropha and castor bean. Conserved synteny and one-to-one orthologous relationship were observed between jatropha and castor bean $R b o h$ genes. Although exon-intron structures are usually highly conserved between orthologs, loss of certain introns was observed for $J c R b o h B, J c R b o h D$, and $R c R b o h N$, supporting their divergence. Global gene expression profiling revealed diverse patterns of $J c R b o h s$ over various tissues. Moreover, expression patterns of $J c R b o h s$ during flower development as well as various stresses were also investigated. These findings will not only improve our knowledge on species-specific evolution of the Rboh gene family, but also provide valuable information for further functional analysis of $R b o h$ genes in jatropha. 
1 TITLE:

\section{Genomics analysis of genes encoding respiratory burst oxidase homologs}

3 (RBOHs) in jatropha and the comparison with castor bean

4

5

6 AUTHORS:

7 Yongguo Zhao ${ }^{1,2}$, Zhi Zou ${ }^{2}$

8

9

10

11

12

13

14

15

21

22 23

24

\section{SHORT TITLE: \\ SHORT TITLE:}

\section{E-MAIL ADDRESSES:}

\section{AFFILIATIONS:}

zhaoyongguo@gdupt.edu.cn (Y.G Zhao), zouzhi2008@126.com or zouzhi@itbb.org.cn (Z.Zou)

${ }^{1}$ Guangdong University of Petrochemical Technology, Maoming 525000, Guangdong, P. R. China

${ }^{2}$ Hainan Key Laboratory for Biosafety Monitoring and Molecular Breeding in Off-Season Reproduction Regions, Key Laboratory of Biology and Genetic Resources of Tropical Crops, Ministry of Agriculture and Rural Affairs, Institute of Tropical Biosciences and Biotechnology, Chinese Academy of Tropical Agricultural Sciences, Haikou 571101, Hainan, P. R. China

To whom correspondence should be addressed: Zhi Zou, zouzhi2008@126.com or zouzhi@itbb.org.cn

Insights into jatropha Rboh family genes 


\section{ABSTRACT}

28 Respiratory burst oxidase homologs (RBOHs), which catalyze the production of superoxide from oxygen and

29 NADPH, play key roles in plant growth and development, hormone signaling, and stress responses. Compared

30 with extensive studies in model plants arabidopsis and rice, little is known about RBOHs in other species. This

31 study presents a genome-wide analysis of Rboh family genes in jatropha (Jatropha curcas) as well as the

32 comparison with castor bean (Ricinus communis), another economically important non-food oilseed crop of the

33 Euphorbiaceae family. The family number of seven members identified from the jatropha genome is equal to

34 that present in castor bean, and further phylogenetic analysis assigned these genes into seven groups named

35 RBOHD, - C, - B, -E, -F, -N, and -H. In contrast to a high number of paralogs present in arabidopsis and rice

36 that experienced several rounds of recent whole-genome duplications, no duplicate was identified in both

37 jatropha and castor bean. Conserved synteny and one-to-one orthologous relationship were observed between

38 jatropha and castor bean Rboh genes. Although exon-intron structures are usually highly conserved between

39 orthologs, loss of certain introns was observed for $J c R b o h B, J c R b o h D$, and RcRbohN, supporting their

40 divergence. Global gene expression profiling revealed diverse patterns of JcRbohs over various tissues.

41 Moreover, expression patterns of $J c R b o h s$ during flower development as well as various stresses were also

42 investigated. These findings will not only improve our knowledge on species-specific evolution of the $R b o h$

43 gene family, but also provide valuable information for further functional analysis of $R b o h$ genes in jatropha.

\section{INTRODUCTION}

45 Respiratory burst oxidase, first identified in human phagocytic cells, is a key enzyme that catalyzes the 46 production of superoxide from oxygen and NADPH (Sumimoto, 2008). In higher plants, this enzyme is known 47 as respiratory burst oxidase homolog $(\mathrm{RBOH})$, which was characterized with the presence of one N-terminal 48 NADPH_Ox domain, one Ferric reductase like transmembrane component, one FAD-binding domain, one 49 NAD-binding domain, and several calcium-binding EF-hand motifs (Sumimoto, 2008; Kaur et al., 2018).

50 Since the first $\mathrm{RBOH}$ was characterized in rice (Oryza sativa), a growing number of homologs have been 51 identified in plant lineages (Groom et al., 1996; Kaur et al., 2014). Genome-wide surveys have also been 52 performed in several species, e.g. seven members from grape (Vitis vinifera), seven members from strawberry 53 (Fragaria $\times$ ananassa), nine members from rice (Oryza sativa), nine members from cassava (Manihot 
54 esculenta), ten members from arabidopsis (Arabidopsis thaliana), ten members from rubber (Hevea 55 brasiliensis), and 15 members from wheat (Triticum aestivum) (Sagi \& Fluhr, 2006; Cheng et al., 2013; Wang 56 et al., 2013; Hu et al., 2018; Zhang et al., 2018; Zou et al., 2019a). Evidence showed that RBOHs play key 57 roles in growth, development, hormone signaling, and stress responses of plants (Marino et al., 2012; Kaur et 58 al., 2014; Angelos \& Brandizzi, 2018; Wang et al., 2018; Zou et al., 2019a). Taking the well-studied model 59 plant arabidopsis for example, AtRbohD and AtRbohF function not only in disease resistance (Chaouch et al., 60 2012) and salt stress tolerance (Xie et al., 2011), but also are essential for jasmonic acid-induced expression of 61 genes regulated by the MYC2 transcription factor (Maruta et al., 2011). AtRbohD also participates in endosperm development (Penfield et al., 2006) and ABA-mediated ROS production and stomatal closure (Zhang et al., 2009). Although the activation mechanism of AtRBOHD and AtRBOHF is similar in stress responses, AtRBOHD was shown to have a significantly greater ROS-producing activity than AtRBOHF

65
(Kimura et al., 2013). Analysis of mutants also revealed that AtRbohD is the major constitutively active member, and AtRbohF is a biotic stress-inducible member (Torres et al., 2002). AtRbohB participates in seed after-ripening (Müller et al., 2009) and AtRbohC functions in root-hair-tip growth (Takeda et al., 2008). AtRbohJ and AtRbohH play essential roles in pollen tube tip growth via $\mathrm{Ca}^{2+}$-activated ROS production (Kaya et al., 2014), whereas AtRbohJ also functions in salt stress tolerance (Evans et al., 2005). Compared with extensive studies in arabidopsis, research in other plants is still in its infancy.

Jatropha (also known as physic nut, Jatropha curcas L., $2 n=22$ ) and castor bean (Ricinus communis L., $2 n$ $=20$ ) are two economically important non-food oilseed crops of the Euphorbiaceae family (Zou et al., 2015b, 2018; Zou, 2018; Zou \& Zhang, 2019). Jatropha is characterized as a small tree native to central America, whereas castor bean is a perennial shrub originated from Africa (Zou et al., 2016a, 2016b, 2018). Based on available genome sequences, the number of 27,172 putative protein-coding genes in jatropha is relatively less than 31,221 in castor bean, which is consistent with a smaller genome size of $320 \mathrm{Mb}$ in jatropha than $400 \mathrm{Mb}$ in castor bean (Chan et al., 2010; Wu et al., 2015). According to comparative genomics analysis, these two species didn't experience additional whole-genome duplication (WGD) after the so-called whole-genome triplication gamma $(\gamma)$ event occurred at approximately 117 Mya (million years ago) (Chan et al., 2010; Jiao et al., 2012; Wu et al., 2015). Further analysis revealed that these two species may diverge from a common

Peer] reviewing PDF | (2018:11:33019:2:2:NEW 23 May 2019) 
81 ancestor at about 49.4 Mya (Wu et al., 2015). Despite the importance of RBOHs, little information is available

82 in these two particular species. Herein, we present a genome-wide comparative analysis of the $R b o h$ gene

83 family in jatropha and castor bean, including gene structures, evolutionary relationships, motif distribution, as

84 well as expression profiles with a focus on jatropha. Our findings provide valuable information for further

85 functional analysis of Rboh genes in these two species.

86 MATERIALS \& METHODS

87 Identification and manual curation of $\boldsymbol{R b o h}$ family genes

88 Arabidopsis and rice Rboh genes described before were retrieved from TAIR10 (Lamesch et al., 2012) or 89 RGAP7 (Sakai et al., 2013), respectively, and their accession numbers are shown Supplementary Table S1. $90 \mathrm{At} / \mathrm{OsRBOH}$ proteins were used as queries to search for homologs from jatropha and castor bean genomes that 91 were downloaded from NCBI (NCBI Resource Coordinators, 2018) or Phytozome v12 (Goodstein et al., 92 2012), respectively. Sequences with an E-value of less than 1E-5 in the tBLASTn search (Altschul et al., 1997) 93 were collected and computationally predicted gene models were manually revised with cDNAs, ESTs 94 (expressed sequence tags) and RNA-seq (RNA sequencing) reads that are available in NCBI (last accessed Jan 95 2018). Presence of the conserved NADPH_Ox domain in candidate RBOHs was confirmed using MOTIF 96 Search (http:/www.genome.jp/tools/motif/) and gene structures were displayed using GSDS (Hu et al., 2015).

97 Homology search for nucleotides or ESTs and expression annotation using RNA-seq data were performed as 98 previously described (Zou et al., 2015a, 2015b).

99 Synteny analysis, sequence alignment and phylogenetic analysis

100 Synteny analysis and multiple sequence alignment of deduced RBOH proteins were carried out as previously

101 described (Zou et al., 2017, 2019b). Phylogenetic trees were constructed using MEGA (version 6.0) (Tamura 102 et al., 2013), implementing the maximum likelihood method with a bootstrap of 1,000 replicates. Orthologs 103 were determined using the BRH (Best Reciprocal Hit) method as described before (Zou et al., 2018) and

104 further confirmed using the result from synteny analysis of jatropha and castor bean. Sequence alignment of 105 Jc/RcRBOHs was displayed using Boxshade (http://sourceforge.net/projects/boxshade/). 
107 Various physical and chemical parameters of $\mathrm{RBOH}$ proteins were calculated using ProtParam (Gasteiger et 108 al., 2005) and subcellular localization was predicted using Plant-mPLoc

109 (http://www.csbio.sjtu.edu.cn/bioinf/plant/). Analysis of conserved motifs was performed using MEME

110 (http://meme-suite.org/tools/meme): any number of repetitions distributed in sequences, the maximum number

111 of 15 motifs, and the width of each motif ranging from six to 180 residues.

\section{Gene expression analysis}

113 Various transcriptome data were retrieved from NCBI SRA and detailed information is shown in

114 Supplementary Table S2. Raw reads were first filtered using fastQC

115 (http://www.bioinformatics.babraham.ac.uk/projects/fastqc/), and resulted clean reads were mapped to the 116 coding sequences (CDS) of revised JcRbohs or RcRbohs as well as other protein-coding genes using Bowtie 2 117 (Langmead \& Salzberg, 2012), and the relative transcript level was represented by FPKM (fragments per 118 kilobase of exon per million fragments mapped, for pair-ended samples) or RPKM (Reads per kilobase per 119 million mapped reads, for single-ended samples) (Mortazavi et al., 2008; Trapnell et al., 2010). The 120 significance of gene expression difference was determined with parameters "FDR $<0.001$ " and " $\log 2$ Ratio $\geq 1$ " 121 with our in-house scripts. Unless specific statements, the tools used in this study were performed with default 122 parameters.

123 RESULTS

\section{Characterization of seven $R$ Roh family genes in jatropha}

125 A survey of the jatropha genome resulted in seven loci that were proven to encode Rboh genes. Considering 126 the extensively functional analysis performed in arabidopsis, JcRbohB, JcRbohC, JcRbohD, JcRbohE, $127 J_{c R b o h F}$, and $J_{c R b o h H}$ were named after their best orthologs in arabidopsis, whereas $J c R b o h N$ represents a 128 novel group member with no ortholog in arabidopsis as well as rice (see below). Based on the result of mining 129 RNA-seq data, all these genes were shown to be expressed. Moreover, $J c R b o h C, J c R b o h D$, and $J c R b o h F$ have 130 corresponding ESTs in NCBI GenBank (Table 1). Compared with the original genome annotation, one gene 131 model was manually optimized on the basis of read alignment: the locus JCGZ_22480 (JcRbohN) was 132 predicted to contain 14 introns putatively encoding 755 residues (KDP26234) and actually it only contains 13 
133 introns encoding 736 residues (see Supplementary File S1). Based on available genetic markers ( $W u$ et al.,

134 2015), six Rboh-encoding scaffolds were further anchored to five out of the 11 chromosomes (Chrs) (Fig. 1).

\section{Characterization of seven $R b o h$ family genes in castor bean}

136 To facilitate evolutionary analysis, Rboh family genes present in castor bean were also identified, resulting in

137 seven genes that are also distributed across six scaffolds. RcRbohB and RcRbohD are located on the same

138 scaffold, which is similar to JcRbohB and $J c R b o h D$ as observed in jatropha (Table 1), corresponding to the

139 close phylogenetic relationship between these two Euphorbiaceous plants. A significant level of syntenic

140 relation and one-to-one orthologous relationship were observed between jatropha and castor bean Rboh genes,

141 supporting their divergence before speciation of these two species. As shown in Fig. 1, synteny analysis also

142 allows anchoring all RcRbohs to five jatropha chromosomes. Based on manual curation, three of the

143 computationally predicted gene models were optimized. The locus $30147 . t 000648$ (RcRbohC) was predicted to

144 encode 710 residues (30147.m014377), and it actually represents only the 3 ' sequence of the gene which

145 encodes 913 residues (see Supplementary File S2). The locus 30128.t000051 (RcRbohD) was predicted to

146 harbor 10 introns putatively encoding 709 residues (30128.m008590), which is relatively shorter than its

147 ortholog in jatropha. In fact, $R c R b o h D$ was also proven to harbor 11 introns, though it contains at least two

148 alternative splicing isoforms. The most common isoform encodes 916 residues (see Supplementary File S3),

149 whereas the short isoform (i.e. 30128.m008590) is derived from the skipping of the tenth intron. The locus

$15029941 . t 000003(R c R b o h N)$ was predicted to harbor 12 introns putatively encoding 666 residues

151 (29941.m000220), which is relatively shorter than its ortholog in jatropha. Actually, read alignment indicated

152 that this gene contains 13 introns encoding 731 residues (see Supplementary File S4).

\section{Phylogenetic analysis, exon-intron structures, and conserved motifs}

154 To reveal the evolutionary relationship of Rboh family genes and facilitate the transfer of functional 155 information obtained in model plants, an unrooted phylogenetic tree was constructed from full-length $156 \mathrm{Jc} / \mathrm{Rc} / \mathrm{At} / \mathrm{OsRBOHs}$, where arabidopsis and rice represent the model eudicot or monocot, respectively. As

157 shown in Fig. 2A, these RBOHs were clustered into seven groups named RBOHC, -D, -B, -E, -F, -N, and -H, 158 which is highly consistent with the BRH-based homologous analysis (Table 1). As for both jatropha and castor 159 bean, a single member was found in each group. By contrast, recent duplicates were found in most groups of 
160

161

162

163

164

165

166

167

168

169

170

171

172

173

174

175

176

177

178

179

180

181

182

183

184

185

186

arabidopsis and rice Rboh gene families, which were shown to result from WGD as well as local duplication (see Supplementary Table S1). However, RBOHC is absent from rice, whereas RBOHN is absent from both arabidopsis and rice, suggesting lineage-specific gene loss. The classification is further supported by exonintron structure patterns as well as conserved motifs. As shown in Fig. 2B, RBOHB, RBOHC, and RBOHD feature 11 introns, whereas RBOHF, RBOHE, RBOHH, and RBOHN usually contain 13 introns. However, gene-specific gain or loss of certain introns was also observed, i.e. JcRbohB, JcRbohD, RcRbohN as well as AtRbohC, AtRbohG, AtRbohD, AtRbohH, AtRbohJ, OsRbohH, OsRbohA, and OsRbohD. And the variety of exon-intron structure in both jatropha and castor bean seems to be considerably less frequent than that in arabidopsis and rice. Compared with exon, the intron length of Rboh genes examined in this study is relatively more variable, where the third intron of OsRbohA harbors the maximum of 6,173 bp (Fig. 2B).

Several parameters of deduced $\mathrm{RBOH}$ proteins were also computed as shown in Table 1. JcRBOHs and RcRBOHs consist of 736-953 or 731-940 amino acids (AA), respectively, and the average sequence length of 887 AA in these two species is relatively smaller than 906 AA in arabidopsis or 903 AA in rice. The average theoretical MW (molecular weight) of $100.72 \mathrm{kDa}$ in jatropha and $100.86 \mathrm{kDa}$ in castor bean is similar to $101.55 \mathrm{kDa}$ in rice or $102.93 \mathrm{kDa}$ in arabidopsis. The average $\mathrm{p} I$ (isoelectric point) value of 9.21 in jatropha and 9.11 in castor bean is also similar to 9.20 in arabidopsis or 9.34 in rice. The GRAVY (grand average of hydropathicity) value varies from -0.081 to -0.362 , supporting their hydrophilic feature (Table 1 and Supplementary Table S1). Subcellular localization analysis showed that all examined RBOHs are located to the cell membrane. In addition to six transmembrane $\alpha$-helices containing two hemes, several typical domains such as Ferric_reduct, FAD_binding_8, NAD_binding_6, NADPH_Ox, and EF-hand motif were found in all Jc/RcRBOHs, though Jc/RcRBOHN lack a large part of the N-terminal as observed in other proteins (Fig. 3). In fact, the N-terminal of RBOHs was shown to be relatively variable related to the $\mathrm{C}$-terminal.

$$
\text { Conserved motifs were further analyzed by using MEME as shown in Fig. 2C and Supplementary Fig. S1. }
$$
Among 15 motifs identified, Motifs 1-3, 5-12 are broadly distributed; Motif 4 is only absent from AtRBOHI and OsRBOHF, and Motifs 13-15 are absent from JcRBOHN and RcRBOHN. Moreover, Motif 13 is also absent from AtRBOHB and OsRBOHA; Motif 14 is also absent from AtRBOHC, AtRBOHA, AtRBOHG, AtRBOHD, OsRBOHD, and OsRBOHA; and, Motif 15 is also absent from AtRBOHA, AtRBOHI, AtRBOHE, 
187 OsRBOHF, and OsRBOHG. Motifs 13, 14, and 11 are part of the NADPH_Ox domain, which was proven to 188 catalyze superoxide production; Motif 4 includes two EF-hand motifs, which are related to $\mathrm{Ca}^{2+}{ }_{-}$binding and $189 \mathrm{Ca}^{2+}$-dependent phosphorylation; Motifs 3, 12, 8, 9, and 6 are part of the Ferric_reduct domain that is similar 190 to ferric reductase; Motif 1 is characterized as the FAD_binding_8 domain that bears the FAD-binding site; 191 and, Motifs 5, 7, 2, and 10 are part of the NAD_binding_6 domain that bears the NAD-binding site (Fig. 2C).

\section{Expression profiles of JcRboh genes}

193 Despite the expression of all JcRbohs, the transcript of $J c R b o h H$ was only detected in late stages of ovule and 194 stamen development as well as a mixed sample of root, mature leaf, flower, developing seed, and embryo 195 (Natarajan \& Parani, 2011; Hui et al., 2017), and the transcript level was shown to be extremely low. A 196 global view of $J c R b o h$ expression profiles was further investigated based on transcriptomes of several typical 197 tissues, i.e. roots from 15-day-old seedlings, half expanded and mature leaves from 4-year-old plants, 198 developing seeds from fruits harvested 19-28 days after pollination (DAP), undifferentiated inflorescence of $1990.5 \mathrm{~cm}$ diameter (IND), female flowers with carpel primordia beginning to differentiate (PID1), female flowers 200 with three distinct carpels formed (PID2), male flowers with stamen primordia beginning to differentiate 201 (STD1), and male flowers with ten complete stamens formed (STD2). Results showed that the total family 202 transcripts are most abundant in PID1 (defined as Class I); moderate in IND, seed, STD2, PID2, root, and 203 STD1 (defined as Class II, accounting for 36-59\% of Class I); and, relatively low in mature leaf and leafage 204 (defined as Class III, accounting for 21-27\% of Class I). JcRbohC and JcRbohD are constitutively expressed 205 in most tissues examined: $J c R b o h C$ occupies $72 \%, 63 \%, 46 \%, 25 \%, 23 \%$ or $23 \%$ of the total transcripts in 206 seed, mature leaf, root, leafage, and IND, respectively; and, $J c R b o h D$ occupies $62 \%, 50 \%, 49 \%, 42 \%, 38 \%$ or $20724 \%$ in STD2, PID1, STD1, PID2, INDs, and root, respectively. Additionally, JcRbohF occupies $68 \%, 27 \%$ or $20821 \%$ of the total transcripts in leafage, mature leaf, and root, respectively, though its transcript level is 209 extremely low in IND, PID1, PID2, STD1, and STD2. Compared with leafage, JcRbohC is upregulated by 210 about 3.0 folds in mature leaf, whereas $J c R b o h F$ is downregulated by about 2.3 folds. Compared with IND, $211 J c R b o h D$ is upregulated by about 2.2 folds in PID1; JcRbohC is downregulated by about 2.3 folds in PID2; $212 J c R b o h B$ and $J c R b o h C$ are downregulated by about 2.9 or 2.7 folds in STD1, respectively; and, $J c R b o h C$ is 213 downregulated by about 2.2 folds in STD2. Compared with PID1, there are 0.4, 0.5 or 0.4 fold-changes in 


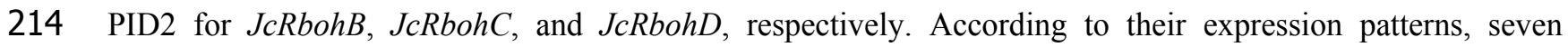

215 JcRbohs could be classed into four main clusters: Cluster I is predominantly expressed in PID1, including



217 Cluster III which includes $J c R b o h F$ is typically expressed in leafage; and, Cluster IV which includes $J c R b o h E$ 218 is mostly expressed in root (Fig. 4).

219 The response to drought and salt stresses was investigated in leaves and roots of 8-week old seedlings as 220 described before (Zhang et al., 2014; Zhang et al., 2015). After withholding irrigation for 1, 4 or 7 d, all $221 J c R b o h s$ were shown to be significantly regulated at least one time point in at least one of the examined tissues.

222 For $1 \mathrm{~d}$, about 3.8-folds downregulation was observed for $J c R b o h D$ in leaf, whereas in root, decrease of about $2232.6,3.9$, and 2.2 folds was observed for $J_{c} R b o h C, J_{c} R b o h N$ or $J_{c} R b o h E$, respectively. For $4 \mathrm{~d}$, about 4.8 -folds 224 decrease was observed for $J c R b o h N$ in root, while in leaf, increase of about 2.8 and 23.4 folds was observed 225 for $J c R b o h B$ or $J c R b o h N$, respectively. For $7 \mathrm{~d}$, decrease of 2.0 and 2.2 folds was observed for $J c R b o h D$ or $226 J c R b o h F$ in root, respectively; by contrast, in leaf, increase of about 3.4, 10.7, 399.1 and 5.9 folds was 227 observed for $J_{c} R b o h B, J_{c} R b o h D, J_{c} R b o h N$ or $J_{c} R b o h E$, respectively. After the treatment with $100 \mathrm{mM} \mathrm{NaCl}$ 228 for $2 \mathrm{~h}, 2 \mathrm{~d}$ or $7 \mathrm{~d}$, two genes were significantly regulated (i.e. JcRbohD and $J c R b o h N$ ): for $2 \mathrm{~h}$, 229 downregulation of $J c R b o h D$ (5.1 folds) and $J c R b o h N$ (7.8 folds) was observed in leaf or root, respectively; and 230 upregulation of $J c R b o h N$ in both leaf and root was observed at later two time points, i.e. 5.3 and 12.3 folds for $2312 \mathrm{~d}$ in leaf or root, respectively; and, 8.6 and 8.1 folds for $7 \mathrm{~d}$ in leaf or root, respectively (Fig. 5A).

232 The response to Colletotrichum gloeosporioides in leaf was analyzed on the basis of transcriptomes of two 233 different genotypes, i.e. the susceptible RJ127 and the resistant 9-1. The result showed that JcRbohN was 234 significantly upregulated in leaves of both the susceptible RJ127 and the resistant 9-1, though the fold-change 235 is highly distinct (i.e. 10.4 vs 2.9 folds) (Fig. 5B). Additionally, in the case of RJ127, JcRbohD was 236 significantly induced, whereas $J c R b o h F$ was inhibited (Fig. 5B), implying different regulation mechanism of 237 these two cultivars.

238 Regulation of $J c R b o h s$ by hormones such as gibberellin acid (GA) and 6-benzylaminopurine (BA) (Ni et al., 2392017 ) was also investigated. After application of $10 \mu \mathrm{M}$ BA to young axillary buds for $12 \mathrm{~h}$, the expression of 
$240 J c R b o h N$ increased by about 9.5 folds, by contrast, no such effect was observed for the same concentration of

241 GA (Fig. 5B).

242 DISCUSSION

243 Despite the crucial role of RBOHs in various plant processes (Kaur et al., 2014), little information is available

244 in jatropha and castor bean, two Euphorbiaceous plants of economic importance. The availability of their

245 genome sequences and various transcriptome datasets allows us to analyze this special gene family from a

246 global view. In this study, a genome-wide identification and manual curation were performed, which results in

247 seven Rboh family genes from both species. The family number is same as that present in grape and strawberry,

248 but relatively smaller than other angiosperm plants reported thus far (Sagi \& Fluhr, 2006; Cheng et al., 2013;

249 Wang et al., 2013; Chang et al., 2016; Hu et al., 2018; Zhang et al., 2018; Zou et al., 2019a). The result is

250 consistent with the fact that both jatropha and castor bean didn't experience any recent WGD, which act as a

251 main force for expansion of genes or gene families (Qiao et al., 2019; Zou et al., 2016a, 2017, 2019a,

252 2019b; Zou \& Yang, 2019a, 2019b, 2019c).

253 Phylogenetic and BRH-based analyses were adopted to divide these genes into seven groups, i.e. RBOHD, -

$254 \mathrm{C},-\mathrm{B},-\mathrm{E},-\mathrm{F},-\mathrm{H}$, and -N. The former six groups are also present in arabidopsis and/or rice, whereas RBOHN

255 represents a recently reported group (Zou et al., 2019a). When taking advantage of $O s / A t / J c / R c R b o h s$ to

256 analyze their orthologs in genome-sequenced plants available in Phytozome and other public databases, we

257 found that RBOHE is relatively primitive, which is also present in Physcomitrella patens, a basal lineage of

258 land plants without well-developed vasculature (Rensing et al., 2008). RBOHD is more likely to first appear in

259 ancient seed plants, since it is present in gymnosperms but not in Selaginella moellendorffii, a member of an

260 ancient vascular lineage (Banks et al., 2011). Both RBOHH and RBOHN can be traced back to Amborella

261 trichopoda, a basal angiosperm plant (Amborella Genome Project, 2013). RBOHN, which is absent from

262 arabidopsis and rice, can be found in several dicots as well as monocots, e.g. cassava, rubber, cacao

263 (Theobroma cacao), cucumber (Cucumis sativus), Aquilegia coerulea, banana (Musa acuminata), maize (Zea

264 mays), and sorghum (Sorghum bicolor) (Supplementary Table S3), supporting species/lineage-specific gene

265 loss of this special group. Both RBOHB and RBOHF are widely found in angiosperms but not in A. trichopoda,

266 suggesting that they may appear sometime before monocot-dicot divergence. RBOHC seems to be the

Peer) reviewing PDF | (2018:11:33019:2:2:NEW 23 May 2019) 
267 youngest one that is widely present in eudicots but not in A. coerulea, a basal eudicot plant (Zou et al., 2018).

268 Thereby, RBOHC may result from the $\gamma$ WGD shared by core eudicots (Jiao et al., 2012). In contrast to 269 jatropha and castor bean that harbor a single member for all seven phylogenetic groups, lineage-specific gain 270 or loss of Rboh family genes was frequently observed in other species, especially those having experienced 271 recent WGDs. For example, four paralogs present in arabidopsis were shown to result from $\beta$ WGD (1), $\alpha$ 272 WGD (2) as well as local duplication (1); one grape duplicate was derived from local duplication; four rice 273 duplicates were all derived from WGD shared by Gramineous plants such as maize, sorghum, millet (Setaria 274 viridis), and foxtail (Setaria italica); two cassava and three rubber duplicates were all derived from the $\rho$ WGD 275 shared by these two species; and, lineage-specific expansion was also observed in banana (Musa acuminata), 276 poplar (Populus trichocarpa), and Brassicaceous plants such as Arabidopsis lyrata, Brassica oleracea, and B. 277 rapa (Supplementary Tables S1 and S3).

278 Although exhibiting one-to-one orthologous relationship and a high level of syntenic relation, divergence of 279 several Rboh genes was observed between jatropha and castor bean. Loss of certain introns was observed for 280 $J c R b o h B, J c R b o h D$, and RcRbohN, though the frequency is relatively lower than that in arabidopsis and rice.

281 Nevertheless, coding sequences are highly conserved and all deduced proteins were shown to contain typical 282 domains essential for superoxide production, though Jc- and RcRBOHN feature a relative shorter N-terminal 283 (Fig. 3).

284 Potential roles of $J_{c} / R c R b o h s$ could be inferred from their expression patterns and function-characterized orthologs in arabidopsis, rice, and other species. According to GO annotation, Rbohs have calcium ion binding, flavin adenine dinucleotide binding, protein binding and superoxide-generating NADPH oxidase activity, and are mainly located to plasma membrane/vacuole that are involved in various biological process as summarized in Supplementary Table S4). Like RcRbohC and RcRbohF (see Supplementary Fig. S2), JcRbohC and $J c R b o h D$ are ubiquitously expressed in most examined tissues of jatropha, supporting their key roles in these tissues. Similar expression patterns were also reported for AtRbohD, AtRbohF, VvRbohA, VvRbohD, OsRbohB, OsRbohA, OsRbohC, OsRbohF, HbRbohC1, HbRbohF2, MeRbohC1, and MeRbohF2 in arabidopsis, rice, 292 grape, cassava, and rubber (Sagi \& Fluhr, 2006; Cheng et al., 2013; Wang et al., 2013; Zou et al., 2019a). 293 Interestingly, $J c R b o h B$ was highly expressed in developing seeds, however, the transcript level of RcRbohB 
294 was shown to be extremely low in seeds, regardless of developing or germinating seeds (Supplementary Fig.

295 S2), supporting their possible function divergence. As reported for AtRbohH and AtRbohJ (Sagi \& Fluhr, 296 2006), both $R c R b o h H$ and $J c R b o h H$ are preferentially expressed in male flowers (Fig. 4 and Supplementary 297 Fig. S2), implying their similar functions. Moreover, the involvement of JcRbohs in flower development and 298 stress responses was also observed. $J c R b o h B, J c R b o h C, J c R b o h D$, and $J c R b o h N$ are highly expressed in five 299 stages of flower development, where $J_{c} R b o h B, J c R b o h C$, and $J_{c R} R o h D$ were shown be significantly regulated 300 between at least two stages. Additionally, $J_{c} R b o h N$, JcRbohD, and $J_{c} R b o h F$ are associated with pathogenic 301 infection, where $J c R b o h N$ is also involved in BA response. In developing castor bean seeds cultured in vitro, 302 transcriptome profiling also showed that RcRbohE and RcRbohH were significantly downregulated by ABA 303 (Chandrasekaran et al., 2014). Thus far, the involvement in pathogenic infection and stress responses has been 304 reported for various Rboh genes in other species, including AtRbohD, AtRbohF, AtRbohJ, OsRbohF1, 305 OsRbohH1, OsRbohE1, VvRbohD1, VvrbohD2, VvRbohF, VvRbohB, VvRbohC, VvRbohH, HbRbohB, 306 HbRbohC1, HbRbohC2, HbRbohD1, HbRbohD2, HbRbohF1, HbRbohF2, and HbRbohN (Evans et al., 2005;

307 Sagi \& Fluhr, 2006; Maruta et al., 2011; Xie et al., 2011; Chaouch et al., 2012; Cheng et al., 2013; Wang et 308 al., 2013; Kaur et al., 2014; Chang et al., 2016; Zou et al., 2019a). More recently, the RBOHF2 in barley was 309 shown to be involved in salicylic acid accumulation and powdery mildew resistance (Torres et al., 2017).

\section{CONCLUSION}

311 To the best of our knowledge, this is the first genome-wide comparative evolutionary analysis of Rboh family genes in jatropha and castor bean. The family number of seven members is relatively smaller than that reported

313 in most angiosperm plants, reflecting no recent WGD occurred in these two special species. Nevertheless, the

314 family is highly diverse, and seven phylogenetic or orthologous groups were found. Among them, RBOHN, a 315 novel but ancient group, was shown to have been lost in many lineages. In jatropha, JcRbohN was shown to 316 widely participate in flower development, hormone signaling, pathogenic infection, and various abiotic stress 317 responses. Additionally, conserved synteny and one-to-one orthologous relationship were observed between $318 J$ JRbohs and RcRbohs, though gene-specific loss of certain introns was observed for several members. These 319 findings will not only improve our knowledge on species-specific evolution of the Rboh gene family, but also 320 facilitate further functional analysis of Rboh genes in jatropha and species beyond. 


\section{ACKNOWLEDGEMENTS}

322 The authors appreciate those contributors who make the related genome and transcriptome data accessible in 323 public databases. They also thank the editor and two reviewers for their helpful suggestions.

\section{Supplemental Information}

325 Supplemental information for this article can be found online at http://dx.doi.org/. 


\section{REFERENCES}

Altschul SF, Madden TL, Schaffer AA, Zhang J, Zhang Z, Miller W, Lipman DJ. 1997. Gapped BLAST and PSI-BLAST: a new generation of protein database search programs. Nucleic Acids Research 25(17):3389-3402 DOI 10.1093/nar/25.17.3389.

Amborella Genome Project. 2013. The Amborella genome and the evolution of flowering plants. Science 342(6165):1241089 DOI: $10.1126 /$ science. 1241089.

Angelos E, Brandizzi F. 2018. NADPH oxidase activity is required for ER stress survival in plants. Plant Journal 96(6):1106-1120 DOI: 10.1111/tpj.14091.

Banks JA, Nishiyama T, Hasebe M, Bowman JL, Gribskov M, dePamphilis C, Albert VA, Aono N, Aoyama T, Ambrose BA, Ashton NW, Axtell MJ, Barker E, Barker MS, Bennetzen JL, Bonawitz ND, Chapple C, Cheng C, Correa LG, Dacre M, DeBarry J, Dreyer I, Elias M, Engstrom EM, Estelle M, Feng L, Finet C, Floyd SK, Frommer WB, Fujita T, Gramzow L, Gutensohn M, Harholt J, Hattori M, Heyl A, Hirai T, Hiwatashi Y, Ishikawa M, Iwata M, Karol KG, Koehler B, Kolukisaoglu U, Kubo M, Kurata T, Lalonde S, Li K, Li Y, Litt A, Lyons E, Manning G, Maruyama T, Michael TP, Mikami K, Miyazaki S, Morinaga S, Murata T, Mueller-Roeber B, Nelson DR, Obara M, Oguri Y, Olmstead RG, Onodera N, Petersen BL, Pils B, Prigge M, Rensing SA, Riaño-Pachón DM, Roberts AW, Sato Y, Scheller HV, Schulz B, Schulz C, Shakirov EV, Shibagaki N, Shinohara N, Shippen DE, Sørensen I, Sotooka R, Sugimoto N, Sugita M, Sumikawa N, Tanurdzic M, Theissen G, Ulvskov P, Wakazuki S, Weng JK, Willats WW, Wipf D, Wolf PG, Yang L, Zimmer AD, Zhu Q, Mitros T, Hellsten U, Loqué D, Otillar R, Salamov A, Schmutz J, Shapiro H, Lindquist E, Lucas S, Rokhsar D, Grigoriev IV. 2011. The Selaginella genome identifies genetic changes associated with the evolution of vascular plants. Science 332(6032):960-963 DOI: $10.1126 /$ science. 1203810 .

Chan AP, Crabtree J, Zhao Q, Lorenzi H, Orvis J, Puiu D, Melake-Berhan A, Jones KM, Redman J, Chen G, Cahoon EB, Gedil M, Stanke M, Haas BJ, Wortman JR, Fraser-Liggett CM, Ravel J, Rabinowicz PD. 2010. Draft genome sequence of the oilseed species Ricinus communis. Nature Biotechnology 28(9):951-956 DOI: $10.1038 /$ nbt.1674.

Chandrasekaran U, Xu W, Liu A. 2014. Transcriptome profiling identifies ABA mediated regulatory changes towards storage filling in developing seeds of castor bean (Ricinus communis L.). Cell Bioscience 4:33 DOI: 10.1186/2045-3701-4-33.

Chang YL, Li WY, Miao H, Yang SQ, Li R, Wang X, Li WQ, Chen KM. 2016. Comprehensive genomic analysis and expression profiling of the NOX gene families under abiotic stresses and hormones in plants. Genome Biology \& Evolution 8(3):791-810 DOI: 10.1093/gbe/evw035.

Chaouch S, Queval G, Noctor G. 2012. AtRbohF is a crucial modulator of defence-associated metabolism and a key actor in the interplay between intracellular oxidative stress and pathogenesis responses in Arabidopsis. Plant Journal 69(4):613-627 DOI: 10.1111/j.1365-313X.2011.04816.x. 
361

362

363

364

365

366

367

368

369

370

371

372

373

374

375

376

377

378

379

380

381

382

383

384

385

386

387

388

389

390

391

392

393

394

395

396

Cheng C, Xu X, Gao M, Li J, Guo C, Song J, Wang X. 2013. Genome-wide analysis of respiratory burst oxidase homologs in grape (Vitis vinifera L.). International Journal of Molecular Sciences 14(12):24169-24186 DOI: 10.3390/ijms 141224169 .

Evans NH, McAinsh MR, Hetherington AM, Knight MR. 2005. ROS perception in Arabidopsis thaliana: the ozone-induced calcium response. Plant Journal 41(4):615-626 DOI: 10.1111/j.1365-313X.2004.02325.x.

Gasteiger E, Hoogland C, Gattiker A, Duvaud S, Wilkins MR, Appel RD, Bairoch A. 2005. Protein Identification and Analysis Tools on the ExPASy Server. (In) John M. Walker (ed): The Proteomics Protocols Handbook, Humana Press, pp. 571-607.

Goodstein DM, Shu S, Howson R, Neupane R, Hayes RD, Fazo J, Mitros T, Dirks W, Hellsten U, Putnam N, Rokhsar DS. 2012. Phytozome: a comparative platform for green plant genomics. Nucleic Acids Research 40(Database issue):D1178-1186 DOI: 10.1093/nar/gkr944.

Groom QJ, Torres MA, Fordham-Skelton AP, Hammond-Kosack KE, Robinson NJ, Jones JD. 1996. rbohA, a rice homologue of the mammalian gp91phox respiratory burst oxidase gene. Plant Journal 10(3):515-522 DOI: 10.1046/j.1365-313X.1996.10030515.x.

Hu B, Jin J, Guo AY, Zhang H, Luo J, Gao G. 2015. GSDS 2.0: an upgraded gene feature visualization server. Bioinformatics 31(8):1296-1297 DOI: 10.1093/bioinformatics/btu817.

Hu CH, Wei XY, Yuan B, Yao LB, Ma TT, Zhang PP, Wang X, Wang PQ, Liu WT, Li WQ, Meng LS, Chen KM. 2018. Genome-wide identification and functional analysis of NADPH oxidase family genes in wheat during development and environmental stress responses. Frontiers in Plant Science 9:906 DOI: 10.3389/fpls.2018.00906.

Hui W, Yang Y, Wu G, Peng C, Chen X, Zayed MZ. 2017. Transcriptome profile analysis reveals the regulation mechanism of floral sex differentiation in Jatropha curcas L. Scientific Reports 7(1):16421 DOI: 10.1038/s41598-017-16545-5.

Jiao Y, Leebens-Mack J, Ayyampalayam S, Bowers JE, McKain MR, McNeal J, Rolf M, Ruzicka DR, Wafula E, Wickett NJ, Wu X, Zhang Y, Wang J, Zhang Y, Carpenter EJ, Deyholos MK, Kutchan TM, Chanderbali AS, Soltis PS, Stevenson DW, McCombie R, Pires JC, Wong GK, Soltis DE, Depamphilis CW. 2012. A genome triplication associated with early diversification of the core eudicots. Genome Biology 13(1):R3 DOI: 10.1186/gb-2012-13-1-r3.

Kaur G, Guruprasad K, Temple BRS, Shirvanyants DG, Dokholyan NV, Pati PK. 2018. Structural complexity and functional diversity of plant NADPH oxidases. Amino Acids 50(1):79-94 DOI: 10.1007/s00726-017-24915.

Kaur G, Sharma A, Guruprasad K, Pati PK. 2014. Versatile roles of plant NADPH oxidases and emerging concepts. Biotechnology advances 32(3):551-563 DOI: 10.1016/j.biotechadv.2014.02.002.

Kaya H, Nakajima R, Iwano M, Kanaoka MM, Kimura S, Takeda S, Kawarazaki T, Senzaki E, Hamamura Y, Higashiyama T, Takayama S, Abe M, Kuchitsu K. 2014. $\mathrm{Ca}^{2+}$-activated reactive oxygen species production by Arabidopsis RbohH and RbohJ is essential for proper pollen tube tip growth. The Plant Cell

Peer) reviewing PDF | (2018:11:33019:2:2:NEW 23 May 2019) 
397

398

399

400

401

402

403

404

405

406

407

408

409

410

411

412

413

414

415

416

417

418

419

420

421

422

423

424

425

426

427

428

429

430

431

432

26(3):1069-1080 DOI: 10.1105/tpc.113.120642.

Kimura S, Kawarazaki T, Nibori H, Michikawa M, Imai A, Kaya H, Kuchitsu K. 2013. The CBL-interacting protein kinase CIPK26 is a novel interactor of Arabidopsis NADPH oxidase AtRbohF that negatively modulates its ROS-producing activity in a heterologous expression system. Journal of Biochemistry 153(2):191-195 DOI: 10.1093/jb/mvs132.

Lamesch P, Berardini TZ, Li D, Swarbreck D, Wilks C, Sasidharan R, Muller R, Dreher K, Alexander DL, Garcia-Hernandez M, Karthikeyan AS, Lee CH, Nelson WD, Ploetz L, Singh S, Wensel A, Huala E. 2012. The Arabidopsis Information Resource (TAIR): improved gene annotation and new tools. Nucleic Acids Research 40(Database issue):D1202-1210 DOI: 10.1093/nar/gkr1090.

Langmead B, Salzberg SL. 2012. Fast gapped-read alignment with Bowtie 2. Nature Methods 9(4):357-359 DOI 10.1038/nmeth.1923.

Marino D, Dunand C, Puppo A, Pauly N. 2012. A burst of plant NADPH oxidases. Trends in Plant Science 17(1):9-15 DOI: 10.1016/j.tplants.2011.10.001.

Maruta T, Inoue T, Tamoi M, Yabuta Y, Yoshimura K, Ishikawa T, Shigeoka S. 2011. Arabidopsis NADPH oxidases, AtRbohD and AtRbohF, are essential for jasmonic acid-induced expression of genes regulated by MYC2 transcription factor. Plant Science 180(4):655-660 DOI: 10.1016/j.plantsci.2011.01.014.

Mortazavi A, Williams BA, McCue K, Schaeffer L, Wold B. 2008. Mapping and quantifying mammalian transcriptomes by RNA-Seq. Nature Methods 5(7):621-628 DOI: 10.1038/nmeth.1226.

Müller K, Carstens AC, Linkies A, Torres MA, Leubner-Metzger G. 2009. The NADPH-oxidase AtRbohB plays a role in Arabidopsis seed after-ripening. New Phytologist 184(4):885-897 DOI: 10.1111/j.14698137.2009.03005.x.

Natarajan P, Parani M. 2011. De novo assembly and transcriptome analysis of five major tissues of Jatropha curcas L. using GS FLX titanium platform of 454 pyrosequencing. BMC Genomics 12:191 DOI: 10.1186/1471-2164-12-191.

NCBI Resource Coordinators. 2018. Database resources of the National Center for Biotechnology Information. Nucleic Acids Research 46(D1):D8-D13 DOI: 10.1093/nar/gkx1095.

Ni J, Zhao ML, Chen MS, Pan BZ, Tao YB, Xu ZF. 2017. Comparative transcriptome analysis of axillary buds in response to the shoot branching regulators gibberellin A3 and 6-benzyladenine in Jatropha curcas. Scientific Reports 7(1):11417 DOI: 10.1038/s41598-017-11588-0.

Penfield S, Li Y, Gilday AD, Graham S, Graham IA. 2006. Arabidopsis ABA INSENSITIVE4 regulates lipid mobilization in the embryo and reveals repression of seed germination by the endosperm. The Plant Cell 18(8):1887-1899 DOI: 10.1105/tpc.106.041277.

Qiao X, Li Q, Yin H, Qi K, Li L, Wang R, Zhang S, Paterson AH. 2019. Gene duplication and evolution in recurring polyploidization-diploidization cycles in plants. Genome Biology 20(1):38 DOI: 10.1186/s13059-019$1650-2$.

Rensing SA, Lang D, Zimmer AD, Terry A, Salamov A, Shapiro H, Nishiyama T, Perroud PF, Lindquist EA,

Peer] reviewing PDF | (2018:11:33019:2:2:NEW 23 May 2019) 
433

434

435

436

437

438

439

440

441

442

443

444

445

446

447

448

449

450

451

452

453

454

455

456

457

458

459

460

461

462

463

464

465

466

467

468

Kamisugi Y, Tanahashi T, Sakakibara K, Fujita T, Oishi K, Shin-I T, Kuroki Y, Toyoda A, Suzuki Y, Hashimoto S, Yamaguchi K, Sugano S, Kohara Y, Fujiyama A, Anterola A, Aoki S, Ashton N, Barbazuk WB, Barker E, Bennetzen JL, Blankenship R, Cho SH, Dutcher SK, Estelle M, Fawcett JA, Gundlach H, Hanada K, Heyl A, Hicks KA, Hughes J, Lohr M, Mayer K, Melkozernov A, Murata T, Nelson DR, Pils B, Prigge M, Reiss B, Renner T, Rombauts S, Rushton PJ, Sanderfoot A, Schween G, Shiu SH, Stueber K, Theodoulou FL, Tu H, Van de Peer Y, Verrier PJ, Waters E, Wood A, Yang L, Cove D, Cuming AC, Hasebe M, Lucas S, Mishler BD, Reski R, Grigoriev IV, Quatrano RS, Boore JL. 2008. The Physcomitrella genome reveals evolutionary insights into the conquest of land by plants. Science 319(5859):64-69 DOI: 10.1126/science.1150646.

Sagi M, Fluhr R. 2006. Production of reactive oxygen species by plant NADPH oxidases. Plant Physiology 141(2):336-340 10.2307/20205750.

Sakai H, Lee SS, Tanaka T, Numa H, Kim J, Kawahara Y, Wakimoto H, Yang CC, Iwamoto M, Abe T, Yamada Y, Muto A, Inokuchi H, Ikemura T, Matsumoto T, Sasaki T, Itoh T. 2013. Rice Annotation Project Database (RAP-DB): an integrative and interactive database for rice genomics. Plant Cell \& Physiology 54(2):e6 DOI: 10.1093/pcp/pcs183.

Sumimoto H. 2008. Structure, regulation and evolution of Nox-family NADPH oxidases that produce reactive oxygen species. FEBS Journal 275(13):3249-3277 DOI: 10.1111/j.1742-4658.2008.06488.x.

Takeda S, Gapper C, Kaya H, Bell E, Kuchitsu K, Dolan L. 2008. Local positive feedback regulation determines cell shape in root hair cells. Science 319(5867):1241-1244 DOI: 10.1126/science.1152505.

Tamura K, Stecher G, Peterson D, Filipski A, Kumar S. 2013. MEGA6: Molecular Evolutionary Genetics Analysis version 6.0. Molecular Biology \& Evolution 30(12):2725-2729 DOI: 10.1093/molbev/mst197.

Torres MA, Dangl JL, Jones JD. 2002. Arabidopsis gp91 phox homologues AtRbohD and AtRbohF are required for accumulation of reactive oxygen intermediates in the plant defense response. Proceedings of the National Academy of Sciences of the United States of America 99(1):517-522 DOI: 10.1073/pnas.012452499.

Torres DP, Proels RK, Schempp H, Hückelhoven R. 2017. Silencing of RBOHF2 causes leaf age-dependent accelerated senescence, salicylic acid accumulation, and powdery mildew resistance in barley. Molecular plantmicrobe interactions 30(11):906-918 DOI: 10.1094/MPMI-04-17-0088-R.

Trapnell C, Williams BA, Pertea G, Mortazavi A, Kwan G, van Baren MJ, Salzberg SL, Wold BJ, Pachter L. 2010. Transcript assembly and quantification by RNA-Seq reveals unannotated transcripts and isoform switching during cell differentiation. Nature Biotechnology 28(5):511-515 DOI: 10.1038/nbt.1621.

Wang GF, Li WQ, Li WY, Wu GL, Zhou CY, Chen KM. 2013. Characterization of rice NADPH oxidase genes and their expression under various environmental conditions. International Journal of Molecular Sciences 14(5):9440-9458 DOI: 10.3390/ijms14059440.

Wang W, Chen D, Zhang X, Liu D, Cheng Y, Shen F. 2018. Role of plant respiratory burst oxidase homologs in stress responses. Free Radical Research 52(8):826-839 DOI: 10.1080/10715762.2018.1473572.

Wu P, Zhou C, Cheng S, Wu Z, Lu W, Han J, Chen Y, Chen Y, Ni P, Wang Y, Xu X, Huang Y, Song C,

Peer] reviewing PDF | (2018:11:33019:2:2:NEW 23 May 2019) 
469

470

471

472

473

474

475

476

477

478

479

480

481

482

483

484

485

486

487

488

489

490

491

492

493

494

495

496

497

498

499

500

501

502

503

504

Wang Z, Shi N, Zhang X, Fang X, Yang Q, Jiang H, Chen Y, Li M, Wang Y, Chen F, Wang J, Wu G. 2015. Integrated genome sequence and linkage map of physic nut (Jatropha curcas L.), a biodiesel plant. Plant Journal 81(5):810-821 DOI: 10.1111/tpj.12761.

Xie YJ, Xu S, Han B, Wu MZ, Yuan XX, Han Y, Gu Q, Xu DK, Yang Q, Shen WB. 2011. Evidence of Arabidopsis salt acclimation induced by up-regulation of HY1 and the regulatory role of RbohD-derived reactive oxygen species synthesis. Plant Journal 66(2):280-292 DOI: 10.1111/j.1365-313X.2011.04488.x.

Zhang C, Zhang L, Zhang S, Zhu S, Wu P, Chen Y, Li M, Jiang H, Wu G. 2015. Global analysis of gene expression profiles in physic nut (Jatropha curcas L.) seedlings exposed to drought stress. BMC Plant Biology 15:17 DOI: 10.1186/s12870-014-0397-x.

Zhang L, Zhang C, Wu P, Chen Y, Li M, Jiang H, Wu G. 2014. Global analysis of gene expression profiles in physic nut (Jatropha curcas L.) seedlings exposed to salt stress. PLoS One 9(5):e97878 DOI: 10.1371/journal.pone.0097878.

Zhang Y, Zhu H, Zhang Q, Li M, Yan M, Wang R, Wang L, Welti R, Zhang W, Wang X. 2009. Phospholipase dalphal and phosphatidic acid regulate NADPH oxidase activity and production of reactive oxygen species in ABA-mediated stomatal closure in Arabidopsis. The Plant Cell 21(8):2357-2377 DOI: 10.1105/tpc.108.062992.

Zhang Y, Li Y, He Y, Hu W, Zhang Y, Wang X, Tang H. 2018. Identification of NADPH oxidase family members associated with cold stress in strawberry. FEBS Open Bio 8(4):593-605 DOI: 10.1002/22115463.12393.

Zou Z. 2018. Mining gene families in the castor bean genome. In: Rabinowicz $P$, Kole C (Eds.): The Castor Bean Genome, Springer, Switzerland, pp. 135-173.

Zou Z, Gong J, An F, Xie GS, Wang JK, Mo YY, Yang LF. 2015a. Genome-wide identification of rubber tree (Hevea brasiliensis Muell. Arg.) aquaporin genes and their response to ethephon stimulation in the laticifer, a rubber-producing tissue. BMC genomics 16:1001 DOI: 10.1186/s12864-015-2152-6.

Zou Z, Gong J, Huang QX, Mo YY, Yang LF, Xie GS. 2015b. Gene structures, evolution, classification and expression profiles of the aquaporin gene family in castor bean (Ricinus communis L.). PLoS One 10(10):e0141022 DOI: 10.1371/journal.pone.0141022.

Zou Z, Huang QX, Xie GS, Yang LF. 2018. Genome-wide comparative analysis of papain-like cysteine protease family genes in castor bean and physic nut. Scientific Reports 8(1):331 DOI: 10.1038/s41598-017-18760-6.

Zou Z, Xie GS, Yang LF. 2017. Papain-like cysteine protease encoding genes in rubber (Hevea brasiliensis): comparative genomics, phylogenetic and transcriptional profiling analysis. Planta 246(5):999-1018 DOI: 10.1007/s00425-017-2739-z.

Zou Z, Yang LF, Gong J, Mo YY, Wang JK, Cao JH, An F, Xie GS. 2016a. Genome-wide identification of Jatropha curcas aquaporin genes and the comparative analysis provides insights into the gene family expansion and evolution in Hevea brasiliensis. Frontiers in Plant Science 7:395 DOI: 10.3389/fpls.2016.00395.

Zou Z, Yang JH. 2019a. Genomics analysis of the light-harvesting chlorophyll a/b-binding (Lhc) superfamily in

Peer) reviewing PDF | (2018:11:33019:2:2:NEW 23 May 2019) 
505

506

507

508

509

510

511

512

513

514

515

516

517

518

519

520

521

522

523

524

525

526

527

528

529

530

531

532

533

cassava (Manihot esculenta Crantz). Gene 702:171-181 DOI: 10.1016/j.gene.2019.03.071.

Zou Z, Yang JH. 2019b. Genome-wide comparison reveals divergence of cassava and rubber aquaporin family genes after the recent whole-genome duplication. BMC genomics 20, 380 DOI: 10.1186/s12864-019-5780-4.

Zou Z, Yang JH. 2019c. Genomic analysis of Dof transcription factors in Hevea brasiliensis, a rubber-producing tree. Industrial Crops \& Products 134:271-283 DOI: 10.1016/j.indcrop.2019.04.013

Zou Z, Yang JH, Zhang XC. 2019a. Insights into genes encoding respiratory burst oxidase homologs (RBOHs) in rubber tree (Hevea brasiliensis Muell. Arg.). Industrial Crops \& Products 128:126-139 DOI: 10.1016/j.indcrop.2018.11.005.

Zou Z, Yang LF, Wang DH, Huang QX, Mo YY, Xie GD. 2016b. Gene structures, evolution and transcriptional profiling of the WRKY gene family in castor bean (Ricinus communis L.). PLoS One 11(2):e0148243 DOI: 10.1371/journal.pone.0148243.

Zou Z, Zhang XC. 2019. Genome-wide identification and comparative evolutionary analysis of the Dof transcription factor family in physic nut and castor bean. PeerJ 7:e6354 DOI: 10.7717/peerj.6354.

Zou Z, Zhu JL, Zhang XC. 2019b. Genome-wide identification and characterization of the Dof gene family in cassava (Manihot esculenta). Gene 687:298-307 DOI: 10.1016/j.gene.2018.11.053.

\section{Figure and Table legends}

Fig. 1 Chromosomal locations of seven JcRboh genes and their collinear genes in castor bean. Shown are five $R b o h$-encoding chromosomes and the chromosome serial number is indicated at the top. RcRboh genes shown just behind their collinear genes in jatropha are marked in orange.

Fig. 2 Structural and phylogenetic analysis of Rboh family genes in jatropha, castor bean, arabidopsis, and rice. A. Shown is the unrooted phylogenetic tree resulting from full-length RBOH proteins with MEGA6, where the distance scale denotes the number of amino acid substitutions per site and bootstrap values are indicated on the top of each clade. B. Shown is the graphic representation of exon-intron structures displayed using GSDS. C. Shown is the distribution of conserved motifs among RBOH proteins, where different motifs are represented by different color blocks as indicated at the bottom of the figure and the same color block in different proteins indicates a certain motif.

Fig. 3 Sequence alignment of jatropha and castor bean $\mathrm{RBOH}$ proteins. Identical and similar amino acids are highlighted in black or dark grey, respectively. Histidine residues involved in heme binding are indicated by arrows. Conserved domains such as $\mathrm{NADPH}$ _Ox, EF-hand motif, Ferric_reduct, FAD_binding_8, and 
534 NAD_binding_6 were are indicated by single underlines, whereas putative transmembrane $\alpha$-helices (TMs) are 535 indicated by double underlines.

536 Fig. 4 Expression profiles of JcRboh genes in various tissues or developmental stages. Color scale

537 represents FPKM normalized $\log _{10}$ transformed counts, where navy indicates low expression and firebrick3

538 indicates high expression. (IND: Undifferentiated inflorescence of $0.5 \mathrm{~cm}$ diameter; PID1: female flower with

539 carpel primordia beginning to differentiate; PID2: female flower with three distinct carpels formed; STD1:

540 male flower with stamen primordia beginning to differentiate; STD2: male flower with ten complete stamens

541 formed; Leafage: half expanded leaves; Leaf: mature leaves that have fully expanded)

542 Fig. 5 Expression profiles of JcRboh genes upon various stresses. A. Profiles upon drought or salt treatment,

543 where color scale represents FPKM normalized $\log _{10}$ transformed counts, where navy indicates low expression

544 and firebrick3 indicates high expression. B. $\log _{10}$ transformed fold-changes upon Colletotrichum infection and

$545 \mathrm{BA} / \mathrm{GA}$ treatments.

546 Table 1 Rboh family genes identified in this study. 
Figure 1

Fig. 1 Chromosomal locations of seven JcRboh genes and their collinear genes in castor bean

Shown are five Rboh-encoding chromosomes and the chromosome serial number is indicated at the top. $R c R b o h$ genes shown just behind their collinear genes in jatropha were marked in orange.

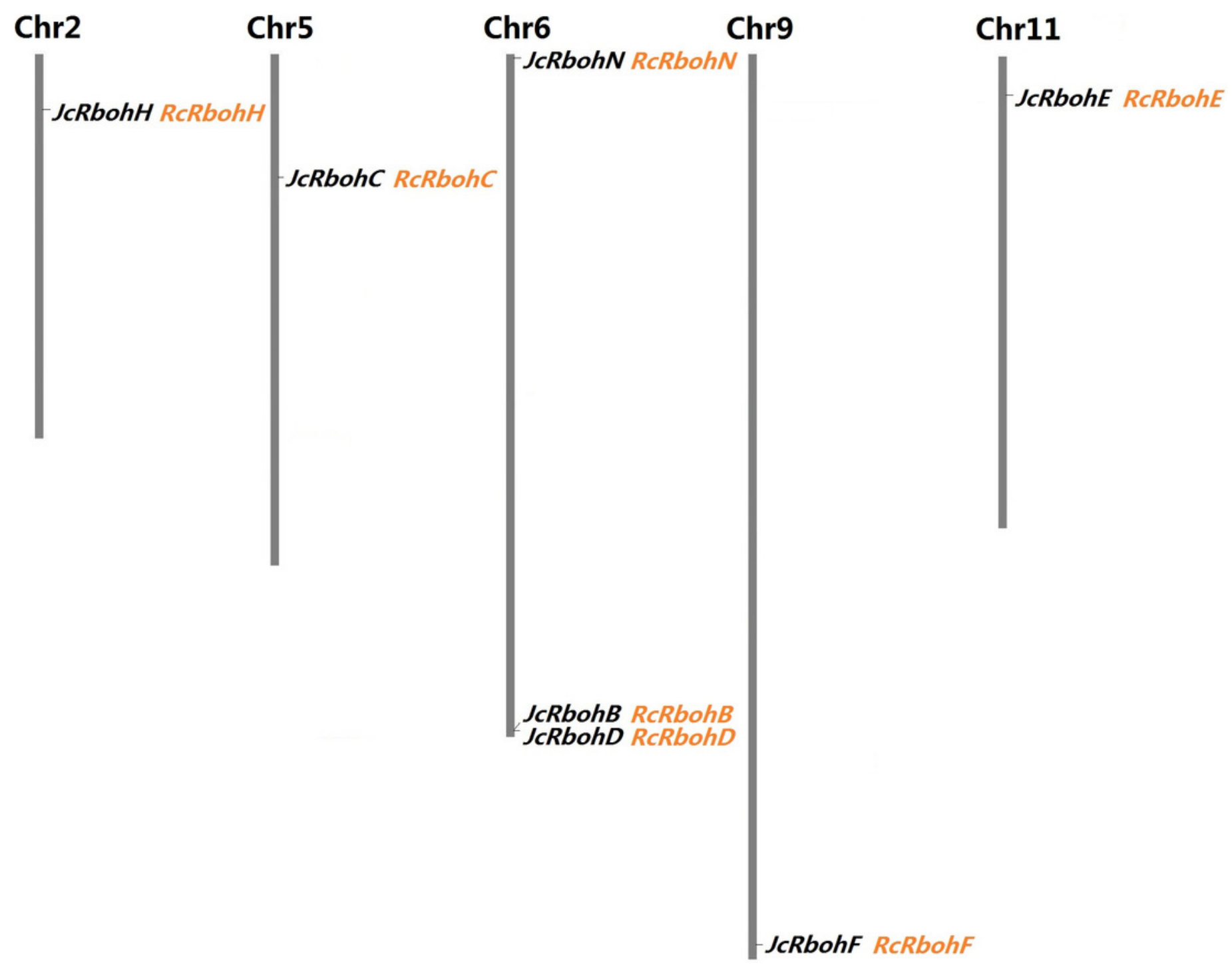


Figure 2

Fig. 2 Structural and phylogenetic analysis of $R$ boh family genes in jatropha, castor bean, arabidopsis, and rice

A. Shown is the unrooted phylogenetic tree resulting from full-length $\mathrm{RBOH}$ proteins with MEGA6, where the distance scale denotes the number of amino acid substitutions per site and bootstrap values are indicated on the top of each clade. B. Shown is the graphic representation of exon-intron structures displayed using GSDS. C. Shown is the distribution of conserved motifs among RBOH proteins, where different motifs are represented by different color blocks as indicated at the bottom of the figure and the same color block in different proteins indicates a certain motif.
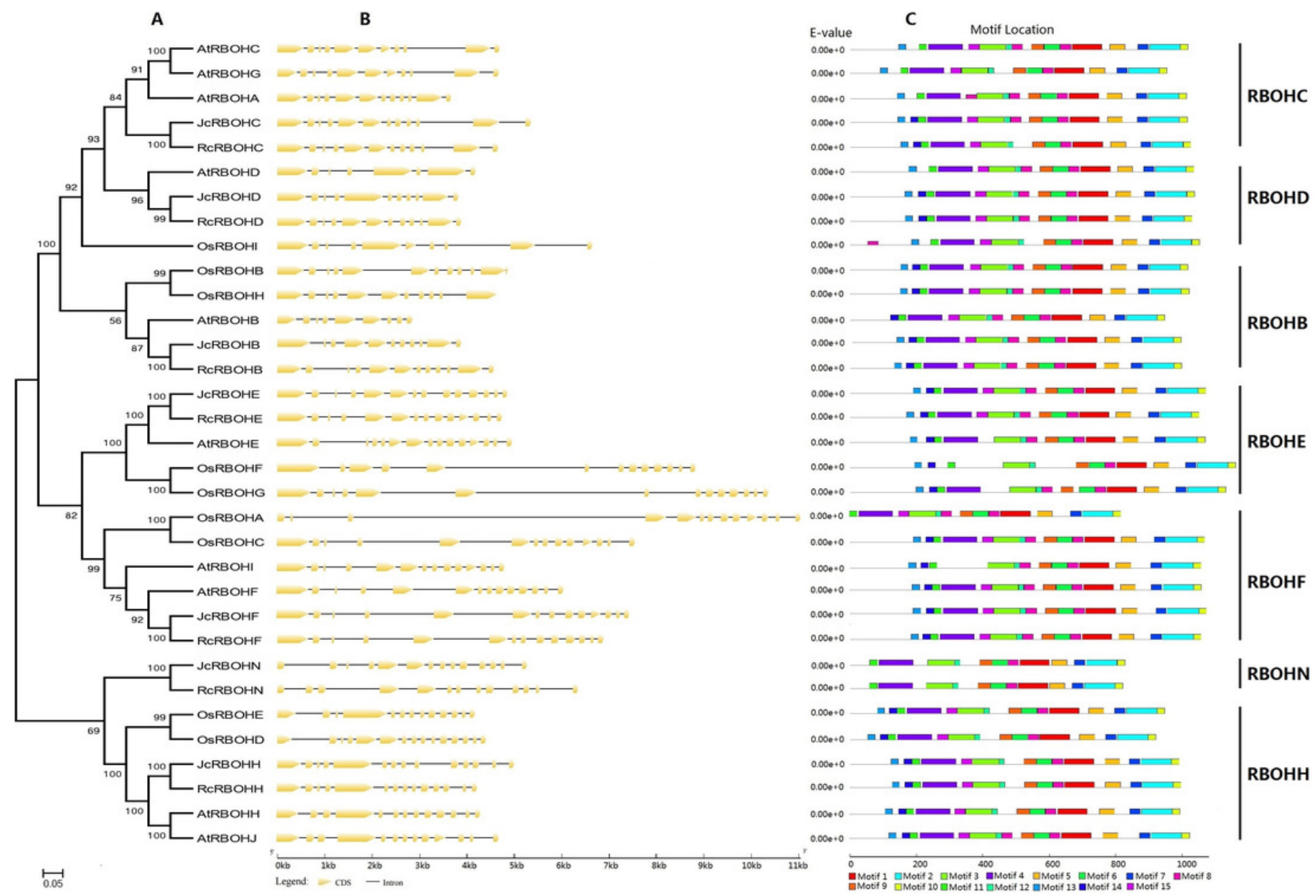


\section{Figure 3}

Fig. 3 Sequence alignment of jatropha and castor bean RBOH proteins

Identical and similar amino acids are highlighted in black or dark grey, respectively. Histidine residues involved in heme binding are indicated by arrows. Conserved domains such as NADPH_Ox, EF-hand motif, Ferric_reduct, FAD_binding_8, and NAD_binding_6 were are indicated by single underlines, whereas putative transmembrane $\alpha$-helices (TMs) are indicated by double underlines. 

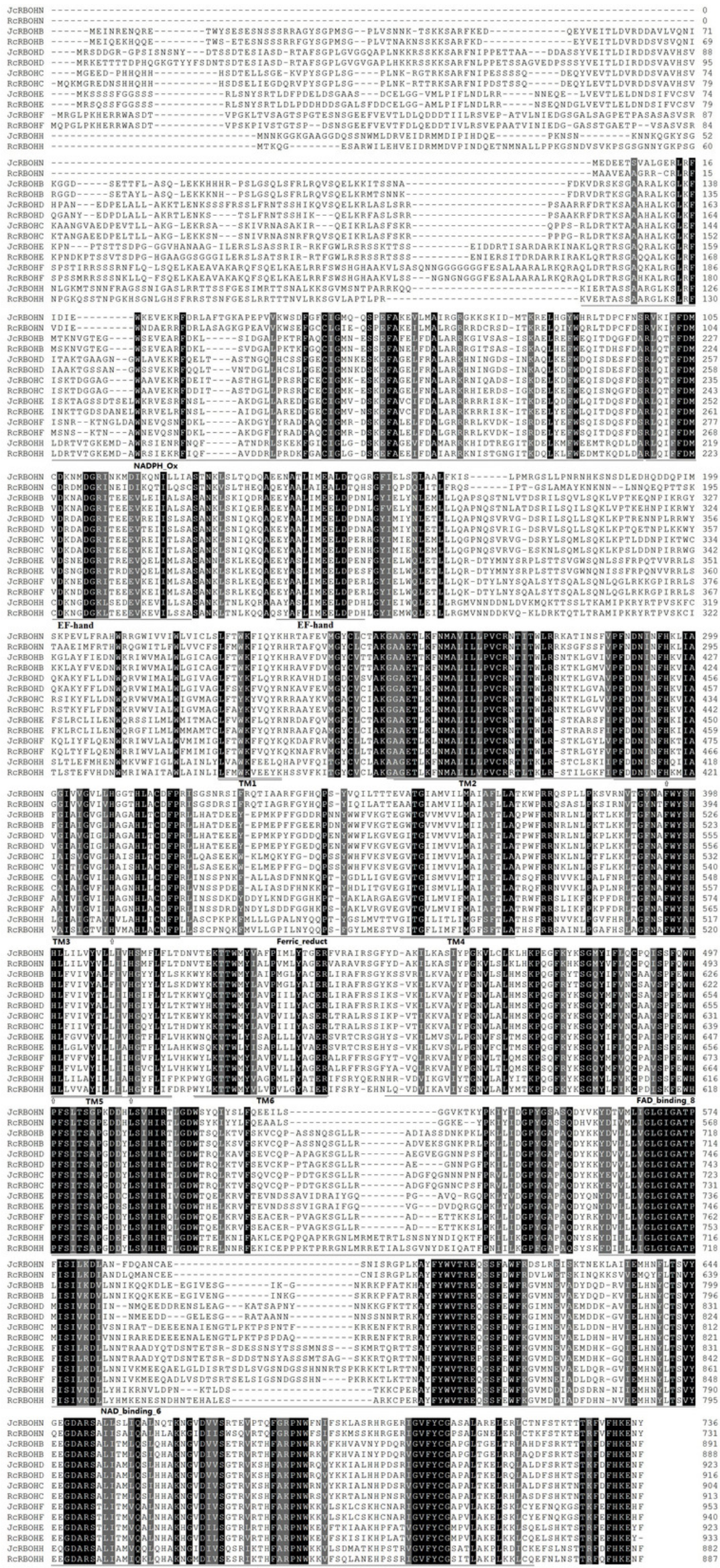

Peer) reviewing PDF | (2018:11:33019:2:2:NEW 23 May 2019) 


\section{Figure 4}

Fig. 4 Expression profiles of $J c R b o h$ genes in various tissues or developmental stages

Color scale represents FPKM normalized $\log _{10}$ transformed counts, where navy indicates low expression and firebrick3 indicates high expression. (IND: Undifferentiated inflorescence of $0.5 \mathrm{~cm}$ diameter; PID1: female flower with carpel primordia beginning to differentiate; PID2: female flower with three distinct carpels formed; STD1: male flower with stamen primordia beginning to differentiate; STD2: male flower with ten complete stamens formed; Leafage: half expanded leaves; Leaf: mature leaves that have fully expanded) 


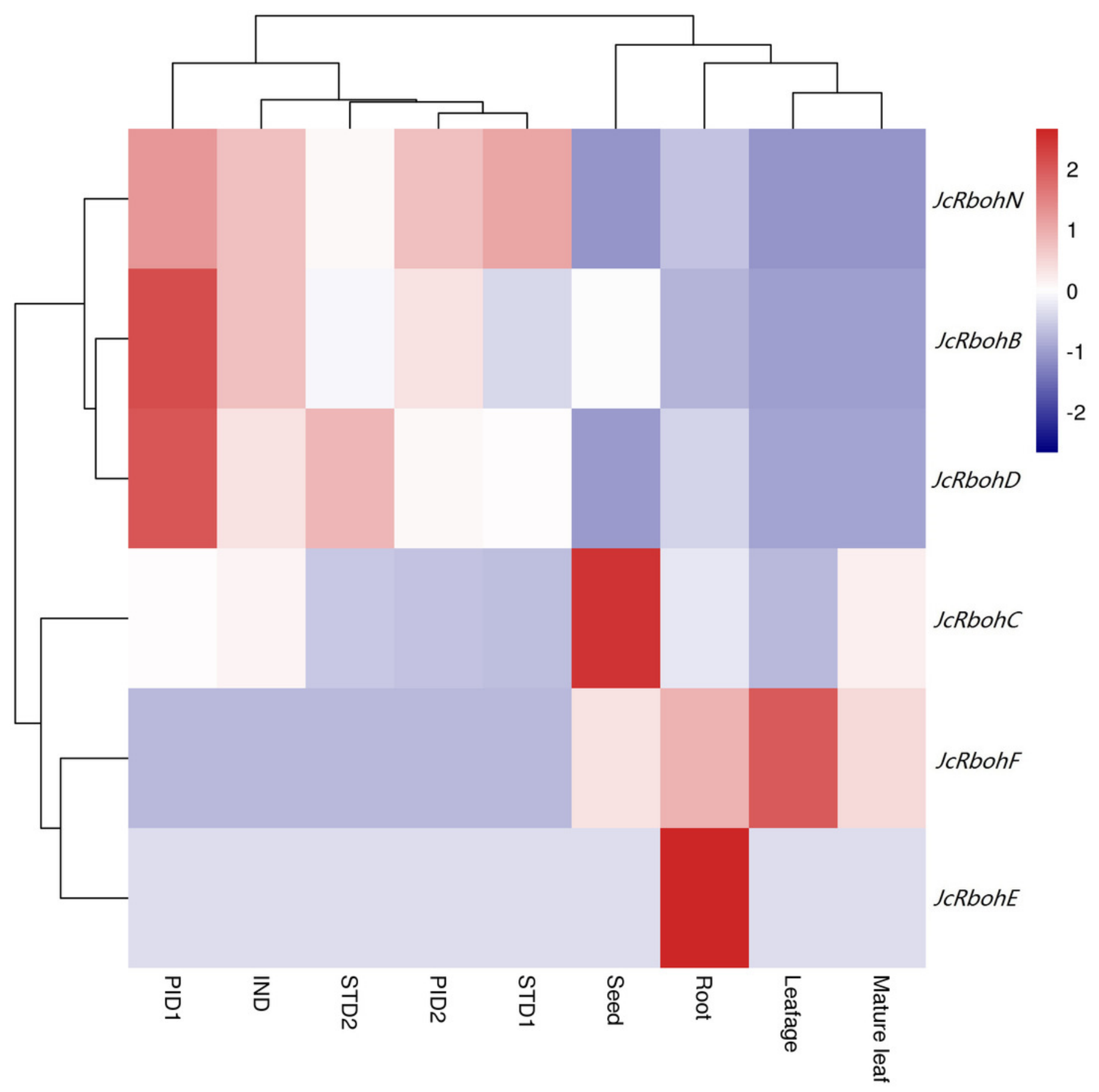


Figure 5

Fig. 5 Expression profiles of $J c R b o h$ genes upon various stresses

A. Profiles upon drought or salt treatment, where color scale represents FPKM normalized $\log _{10}$ transformed counts, where navy indicates low expression and firebrick3 indicates high expression. B. $\log _{10}$ transformed fold-changes upon Colletotrichum infection and BA/GA treatments.
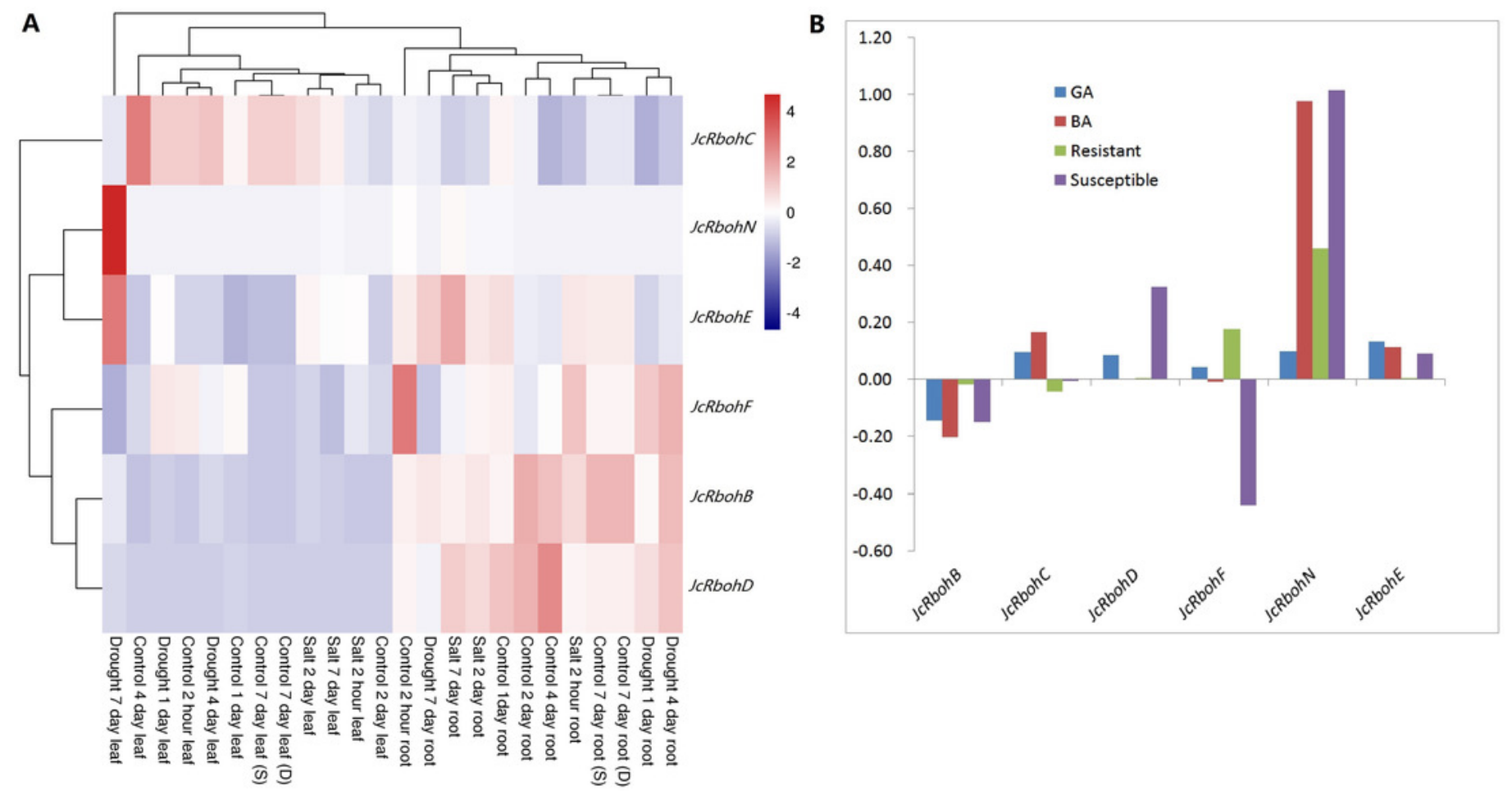


\section{Table $\mathbf{1}$ (on next page)}

Table 1 Rboh family genes identified in this study 


\begin{tabular}{|c|c|c|c|c|c|c|c|c|c|c|c|c|c|}
\hline \multirow[t]{2}{*}{$\begin{array}{l}\text { Gene } \\
\text { name }\end{array}$} & \multirow[t]{2}{*}{ Locus name } & \multirow[t]{2}{*}{ Position } & \multicolumn{2}{|c|}{$\begin{array}{l}\text { Nucleotide length } \\
\text { (bp, from start to } \\
\text { stop codons) }\end{array}$} & \multirow[t]{2}{*}{$\begin{array}{c}\text { Intron } \\
\text { no. }\end{array}$} & \multirow[t]{2}{*}{$\begin{array}{c}\text { EST } \\
\text { no. }\end{array}$} & \multirow[t]{2}{*}{$\mathbf{A A}$} & \multirow[t]{2}{*}{$\begin{array}{l}\text { MW } \\
\text { (kDa) }\end{array}$} & \multirow[t]{2}{*}{$p \mathbf{I}$} & \multirow[t]{2}{*}{$\underset{\mathbf{Y}}{\text { GRAV }}$} & \multirow[t]{2}{*}{$\begin{array}{c}\text { TM } \\
\text { H }\end{array}$} & \multicolumn{2}{|c|}{ Ortholog } \\
\hline & & & CDS & Gene & & & & & & & & At & Os \\
\hline JcRbohC & JCGZ_26338 & scaffold906:2548368-2554294 & 2715 & 5349 & 11 & 5 & 904 & 102.57 & 9.13 & -0.305 & 4 & $\begin{array}{l}\text { AtRbohC } \\
\text { AtRbohA } \\
\text { AtRbohG }\end{array}$ & - \\
\hline$J c R b o h D$ & JCGZ_23064 & scaffold779:96300-100604 & 2772 & 3819 & 10 & 1 & 923 & 103.85 & 9.20 & -0.286 & 4 & AtRbohD & OsRbohI \\
\hline$J c R b o h B$ & JCGZ_23317 & scaffold779:1441569-1437068 & 2676 & 3882 & 10 & 0 & 891 & 101.36 & 9.07 & -0.285 & 4 & AtRbohB & $\begin{array}{l}\text { OsRbohB } \\
\text { OsRbohH }\end{array}$ \\
\hline JcRbohE & JCGZ_07528 & scaffold211:3210360-3215422 & 2772 & 4858 & 13 & 0 & 923 & 104.39 & 8.91 & -0.187 & 4 & AtRbohE & $\begin{array}{l}\text { OsRbohF } \\
\text { OsRbohG }\end{array}$ \\
\hline$J c R b o h F$ & JCGZ_20527 & scaffold660:1101645-8731 & 2862 & 7448 & 13 & 1 & 953 & 108.19 & 9.38 & -0.264 & 4 & $\begin{array}{l}\text { AtRbohF } \\
\text { AtRbohI }\end{array}$ & $\begin{array}{l}\text { OsRbohA } \\
\text { OsRbohC }\end{array}$ \\
\hline JcRbohN & JCGZ_22480 & scaffold729:242504-247775 & 2211 & 5272 & 13 & 0 & 736 & 84.31 & 9.35 & -0.089 & 4 & - & - \\
\hline JcRbohH & JCGZ_05621 & scaffold18:2574685-2569687 & 2649 & 4999 & 13 & 0 & 882 & 100.37 & 9.44 & -0.227 & 6 & $\begin{array}{l}\text { AtRbohH } \\
\text { AtRbohJ }\end{array}$ & $\begin{array}{l}\text { OsRbohE } \\
\text { OsRbohD }\end{array}$ \\
\hline RcRbohC & $30147 . t 000648$ & scaffold30147:1591736-1597026 & 2742 & 4658 & 11 & 0 & 913 & 103.59 & 9.04 & -0.362 & 4 & $\begin{array}{l}\text { AtRbohC } \\
\text { AtRbohA } \\
\text { AtRbohG }\end{array}$ & - \\
\hline RcRbohD & $30128 . t 000051$ & scaffold30128:2636281-2631846 & 2751 & 3885 & 11 & 3 & 916 & 103.17 & 9.04 & -0.257 & 4 & AtRbohD & OsRbohI \\
\hline RcRbohB & 30128.0000279 & scaffold30128:1185430-1190626 & 2667 & 4573 & 11 & 0 & 888 & 101.47 & 9.21 & -0.334 & 4 & AtRbohB & $\begin{array}{l}\text { OsRbohB } \\
\text { OsRbohH }\end{array}$ \\
\hline RcRbohE & $29739 . t 000141$ & scaffold29739:892440-898120 & 2805 & 5681 & 13 & 0 & 934 & 105.82 & 8.78 & -0.239 & 4 & AtRbohE & $\begin{array}{l}\text { OsRbohF } \\
\text { OsRbohG }\end{array}$ \\
\hline RcRbohF & $30190 . t 000521$ & scaffold30190:3095171-3103214 & 2823 & 6880 & 13 & 0 & 940 & 106.96 & 9.32 & -0.259 & 4 & $\begin{array}{l}\text { AtRbohF } \\
\text { AtRbohI }\end{array}$ & $\begin{array}{l}\text { OsRbohA } \\
\text { OsRbohC }\end{array}$ \\
\hline RcRbohN & 29941.0000003 & scaffold29941:28891-17709 & 2196 & 6347 & 12 & 0 & 731 & 83.76 & 9.26 & -0.122 & 5 & - & - \\
\hline RcRbohH & 30039.0000013 & scaffold30039:82700-87109 & 2664 & 4410 & 13 & 0 & 887 & 101.22 & 9.13 & -0.209 & 6 & $\begin{array}{l}\text { AtRbohH } \\
\text { AtRbohJ }\end{array}$ & $\begin{array}{l}\text { OsRbohE } \\
\text { OsRbohD }\end{array}$ \\
\hline
\end{tabular}

1 